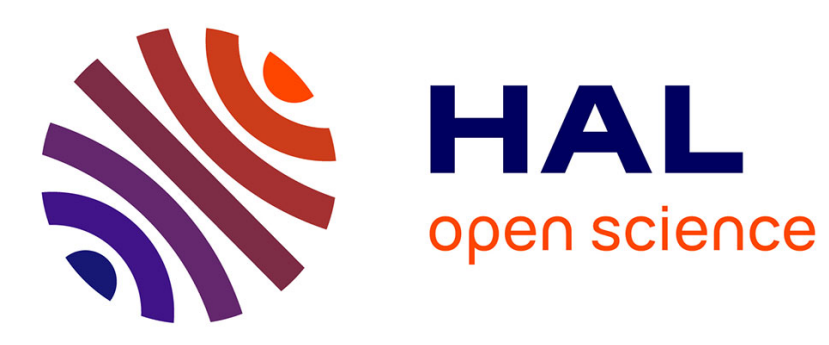

\title{
Faster Diffusion across an Irregular Boundary
}

Anna Rozanova-Pierrat, Denis S Grebenkov, Bernard Sapoval

\section{To cite this version:}

Anna Rozanova-Pierrat, Denis S Grebenkov, Bernard Sapoval. Faster Diffusion across an Irregular Boundary. Physical Review Letters, 2012, 108, pp.240602. 10.1103/PhysRevLett.108.240602 . hal00760296

\section{HAL Id: hal-00760296 \\ https://hal-centralesupelec.archives-ouvertes.fr/hal-00760296}

Submitted on 5 Dec 2012

HAL is a multi-disciplinary open access archive for the deposit and dissemination of scientific research documents, whether they are published or not. The documents may come from teaching and research institutions in France or abroad, or from public or private research centers.
L'archive ouverte pluridisciplinaire HAL, est destinée au dépôt et à la diffusion de documents scientifiques de niveau recherche, publiés ou non, émanant des établissements d'enseignement et de recherche français ou étrangers, des laboratoires publics ou privés. 


\title{
Faster Diffusion across an Irregular Boundary
}

\author{
A. Rozanova-Pierrat \\ Laboratoire de Physique de la Matière Condensée, \\ C.N.R.S. - Ecole Polytechnique, 91128 Palaiseau, France \\ and Laboratoire de Mathematiques Appliquees aux Systemes, \\ Ecole Centrale Paris, 92295 Chatenay-Malabry, France \\ D. S. Grebenkov and B. Sapovat \\ Laboratoire de Physique de la Matière Condensée, \\ C.N.R.S. - Ecole Polytechnique, 91128 Palaiseau, France
}

(Dated: December 5, 2012)

\begin{abstract}
We investigate how the shape of a heat source may enhance global heat transfer at short time. An experiment is described that allows to obtain a direct visualization of heat propagation from a prefractal radiator. We show, both experimentally and numerically, that irregularly-shaped passive coolers rapidly dissipate at short times, but their efficiency decreases with time. The de Gennes' scaling argument is shown to be only a large scale approximation which is not sufficient to describe adequately the temperature distribution close to the irregular frontier. This work shows that radiators with irregular surfaces permits increased cooling of pulsed heat sources.
\end{abstract}

PACS numbers: 05.60.-k, 44.05.+e, 66.10.C-

Propagation of heat as well as particle diffusion are of direct interest in every-day life, chemical industry, life sciences, and pollution spreading. In electronics or civil engineering, one needs to improve the cooling of microprocessors and to build efficient radiators for housing. Empirically, a large surface is known to dissipate more so that irregularly-shaped metallic radiators are placed onto microprocessors in order to remove heat. But this is the result of both conduction and convection. In this letter, we present an experimental, theoretical and numerical study of purely diffusive heat transfer that aims at revealing the role of the shape of the radiator.

This question has been intensively investigated in a steady state regime in which the diffusive transfer towards a boundary is governed by Laplace equation. In this case, a general mathematical result known as the Makarov's theorem states that, in 2D, the information dimension of the harmonic measure (which describes the distribution of particles arrived onto the boundary) is strictly equal to 1 for any simply connected set, whatever the complexity of its boundary [1]. In physical terms, this means that, contrary to intuition, regular and irregular boundaries work similarly in steady state regime [2 [4].

In this letter, we report the first experimental observation that regular and irregular boundaries behave differently in the transitory (or time-dependent) regime. We show that the role of irregular boundaries is important and provide quantitative explanations based on theory and numerical simulations. Note that transitory diffusion from an irregular surface has been already studied in the context of impedance spectroscopy in electrochemistry [5, 6], NMR relaxation in porous media [7, 8], Brownian motion near fractal surfaces $[9]$ and heat transfer [10]. Rigorous short-time asymptotics have been re- ported for diffusive transfer at fractal boundaries 11 17].

We present a direct experimental visualization of heat propagation from a "fractal" radiator in $2 \mathrm{D}$.

Fractals are complex shapes that contain a large variety of geometrical feature sizes [18], and it has been already shown that deterministic and random shapes exhibiting the same fractal dimensions, behave similarly 19.

We check, for the first time, the validity of the de Gennes' scaling argument [20] which is often used to understand heat propagation and diffusion from an irregular surface [5, 7, 21]. Relying on experimental results and numerical solutions of the underlying Fourier equation, we conclude that this argument can only be a large scale approximation which is not sufficient to describe temperature distribution close to the irregular frontier.

The heat propagation in 2D has been studied experimentally using liquid crystal temperature imaging 22, 23]. The heat source is constituted by a highly conductive, $4 \mathrm{~mm}$ thick, aluminum sheet (Fig पa). A complex perforation has been laser machined through this plate to obtain the third generation of the quadratic von Koch snowflake of fractal dimension $3 / 2$. The aluminum heat source is heated around $T_{s} \approx 40^{\circ} \mathrm{C}$. It is then put on the liquid crystal plate (at temperature $T_{0} \approx 20^{\circ} \mathrm{C}$ ) which was set on a Styrofoam block with very small heat conductivity. This constitutes, to a good approximation, a $2 \mathrm{D}$ heat propagation system. A video camera monitors the time evolution of the temperature distribution by following the displacement of the visible isotherm lines imaged by their color (Fig. प(b)-(f)). The entire film can be seen on www... At each time $t$, one can extract by a thresholding procedure an isotherm curve $\mathbf{r}_{t}$ of a given color (at temperature $T$ ). Since a quantitative measure of the heat $Q(t)$ transferred from the source from time 
amount of heat $Q(t)$ transferred into $\Omega$ is simply:

$$
Q(t)=c \delta \int_{\Omega} d \mathbf{r}\left(T(\mathbf{r}, t)-T_{0}\right) .
$$

The fact that Eqs. (2) also describe particle diffusion suggests a random walk interpretation of the conduction experiment. In this representation, a source (here the constant temperature $T_{s}$ at radiator) is maintained at a given concentration. Its boundary emits diffusive random walkers that invade progressively the initially empty medium $\Omega$. These walkers form a diffusion layer whose area can be tentatively used as a proxy for the transferred heat according to Eq. (11).

de Gennes suggested to replace this area by that of the so-called "Minkowski sausage" 27] of average width $l_{D}(t)$ taken of order $\sqrt{K t}$ [20]. The transferred heat is then related, through Eq. (11), to the area $M(t)$ of the Minkowski sausage, $S_{T}(t) \simeq M(t)$, so that the heat diffusion problem is replaced by a purely geometrical computation of the area $M(t)$.

In this frame, a qualitative picture of heat propagation can be drawn from the comparison between the width $l_{D}(t)$ and the relevant geometrical length-scales: the size $l$ of the smallest geometrical detail of the interface (the smaller cut-off) and the size of the larger cutoff $L$ of the boundary. For a prefractal boundary as that of the experiment, three different regimes can be expected:

(i) At very short times, the diffusion width is much smaller than the smallest cut-off $l$ and the whole interface transfers heat (or particles) almost uniformly. The area of the Minkowski sausage is then close to

$$
M(t) \simeq L_{p} \sqrt{K t}
$$

where $L_{p}$ is the total perimeter of the boundary which scales as $L_{p} \propto l(L / l)^{d_{f}}$ for a surface of Minkowski dimension $d_{f}$.

(ii) When the diffusion width $l_{D}$ becomes comparable to $l$, the scaling of the Minkowski sausage behaves differently. For self-similar boundaries, the Minkowski sausage area scales with its width $l_{D}$ as $l_{D}^{d-d_{f}}$, from which [20]:

$$
M(t) \propto L^{d}\left(K t / L^{2}\right)^{\left(d-d_{f}\right) / 2} .
$$

In our case with $d_{f}=3 / 2$ and $d=2$, the transfered heat is expected to follow a $t^{0.25}$ power law. This intermediate regime lasts up to the time when the diffusion length reaches the larger cut-off $L$ of the interface.

(iii) From this time, the heat transfer saturates since the domain is bounded.

Those scaling arguments should provide an explanation for the experimental power laws shown on Fig. 2 And indeed there is a regime where the surface $S_{T}(t)$ follows a power law with an exponent close to 0.25 but it is followed by a regime with an exponent larger that 0.5 .
We show now that the de Gennes argument is not sufficient to interpret the experiment because it neglects the role of confinement which might play an important role depending on the isotherm under consideration. Keeping in mind the exact correspondence with random walkers, one has to take care that the concentration of particles reaching a given point is formed by particles emitted by the several faces surrounding this point. This behavior is indicated by the white arrows in Fig. 1(e), in which the isotherm emerging from a salient wedge is clearly much closer to the surface than the same isotherm in a confined region. In other words, the assumption of an approximately uniform diffusion layer which could be characterized by an average width $l_{D}$, is not valid for our experiment. As a consequence, the two areas, $M(t)$ (fixed by the geometrical width $l_{D}$ of the Minkowski sausage) and $S_{T}(t)$ (set by the isotherm of a given temperature $T)$, are not equivalent.

An exact example illustrating this fact is given in the Supplementary Materials, where we derive the short-time asymptotic behavior of the transferred heat and the area $S_{T}(t)$ for a spherical domain. In two dimensions, we get

$$
\begin{aligned}
Q(t) & \simeq c \delta\left(T_{s}-T_{0}\right) \frac{2}{\sqrt{\pi}} L_{p} \sqrt{K t}, \\
S_{T}(t) & \simeq 2 \beta(T) L_{p} \sqrt{K t}
\end{aligned}
$$

where $L_{p}=2 \pi R$. The factor $\beta(T)$ is given, for spherical domains, by $\beta=\operatorname{erfc}^{-1}(\theta)$, where $\theta=\left(T-T_{0}\right) /\left(T_{s}-T_{0}\right)$ is the relative temperature of the isotherm under consideration $\left(\operatorname{erfc}^{-1}(z)\right.$ is the inverse complementary error function). Although both quantities in Eq. (6) are proportional to $L_{p} \sqrt{K t}$, the quantity associated with the isotherm (i.e, $\left.c \delta\left(T_{s}-T_{0}\right) S_{T}(t)\right)$ differs from the real heat quantity that has diffused. This is due to the value of $\beta$ which can be very small if $\theta$ is close to one, a situation corresponding to the experiment. In particular, the curves shown on Fig. 2 can only be compared up to a shift along $x$ or $y$ axis which represents a multiplication by $\beta$ on logarithmic scale.

In general, the approximation (11) is expected to be more accurate for isotherms with small difference $T-T_{0}$ because nearly all the heat will be found inside the layer. In fact, representing $\Omega$ as $\Omega^{>} \cup \Omega^{<}$(with $\Omega^{\lessgtr}=\{\mathbf{r} \in$ $\Omega: T(\mathbf{r}, t) \lessgtr T\})$, the integral in Eq. (3) splits in two parts which can be approximated at short times as

$$
Q(t) \approx c \delta\left(T_{s}-T\right) \operatorname{Vol}\left(\Omega^{>}\right)+c \delta\left(T-T_{0}\right) \operatorname{Vol}\left(\Omega^{<}\right)
$$

where the first term is close to $c \delta\left(T_{s}-T_{0}\right) S_{T}(t)$ for $T$ close to $T_{0}$, while the second term can be neglected in that case. In the opposite situation, which is relevant for the experiment, the approximation (1) fails.

We illustrate this idea by solving numerically Eqs. (2) for the third generation of the quadratic Von Koch curve shown on Fig. 3] The solution was obtained by the 


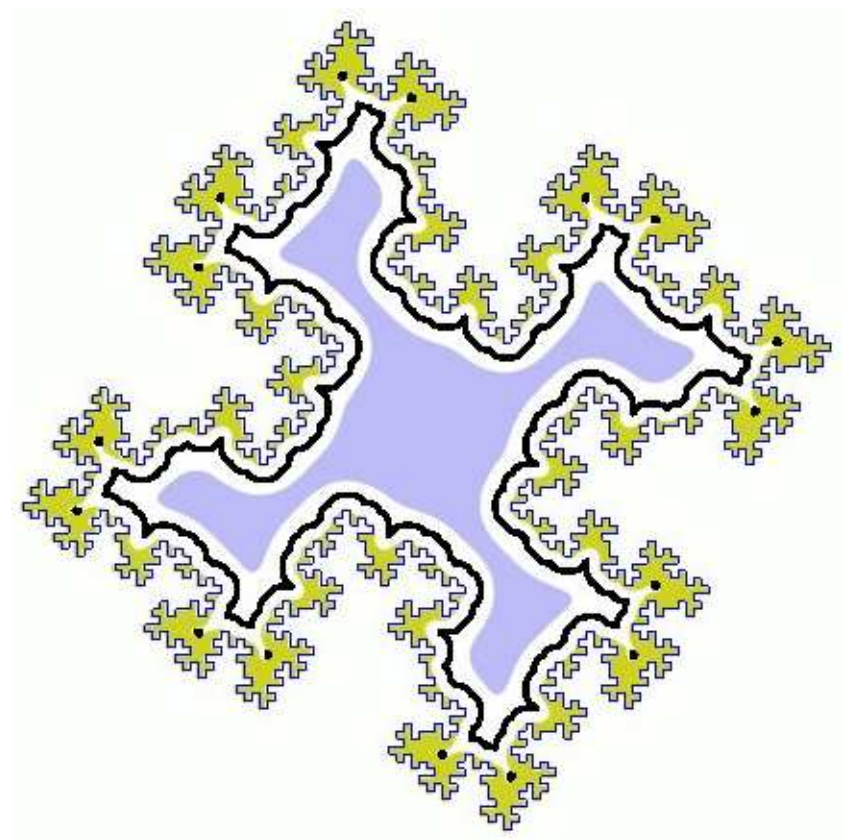

ferred heat (solid curve) indeed exhibits the three scaling regimes discussed above: short-time behavior $\propto t^{0.5}$, intermediate behavior $\propto t^{0.25}$ and finally saturation. The asymptotics are shown by dashed and dash-dotted curves, respectively. (iii) Three normalized surface areas (circles, squares and triangles) have been shifted along the horizontal axis by factors 5,1 and 0.11 in order make them follow the solid curve. These shifts (on logarithmic scale) are equivalent to the choice of the appropriate values for the coefficient $\beta(T)$. As expected, the curve with $\theta=0.1$ shows the best agreement with the solid curve for the transfered heat, while that with $\theta=0.9$ exhibits a strong deviation at long times. (iv) Deviations at short times can be attributed to an inaccurate computation of the surface area $S_{T}(t)$ by the thresholding procedure. Although this computation could be improved for isotherms obtained from numerical simulations, the thresholding procedure remains unavoidable for experimental images. In order to be able to compare numerical and experimental images, we used the same image processing in both cases. (v) The surface area $S_{T}(t)$ estimated from experimental images is also shown on Fig.

FIG. 3: (Color online) Three temperature zones at $t=0.1 \mathrm{~s}$ : $\theta(\mathbf{r}, t)>0.9$ (yellow), $0.1<\theta(r, t)<0.9$ (white) and $\theta(\mathbf{r}, t)<$ 0.1 (blue). The frontiers between successive zones are the isotherm at $\theta=0.9$ (a.u.) and $\theta=0.1$ (a.u.) which are compared to the Minkowski sausage of width $\sqrt{K t}$ (shown by the thick solid line), at $t=0.1 \mathrm{~s}$.

quadratic Lagrange finite differences method in the module "PDE, Coefficient Form" of COMSOL Multiphysics. The time-dependent solver for a symmetric linear system "Spooles" was used. For these simulations, we set: $T_{s}=1$ (a.u.), $T_{0}=0, K=0.01$ (a.u.) and $L=1$ (a.u.) ( $L$ is the base of the snowflake curve). Once the solution $T(\mathbf{r}, t)=\theta(\mathbf{r}, t)$ is found, the isotherm curve $\mathbf{r}_{t}$ was computed by solving $T\left(\mathbf{r}_{t}, t\right)=T$ with a chosen $T$ and then the surface area $S_{T}(t)$ between the isotherm and the boundary was calculated by a thresholding procedure (as for experimental images). Figure 3 shows that, at a given time, the zones defined by the isotherms depend strongly on their temperature. The relative positions of isotherms at a given time illustrate the role of confinement, observed in the experiment (Fig. प).

The total transferred heat $Q(t)$ is also obtained by integrating the solution $T(\mathbf{r}, t)$ over $\Omega$. The results are summarized in Fig. 20 which shows the normalized transferred heat, $Q(t) / Q(\infty)$, and the normalized surface areas, $S_{T}(t) / S_{\text {tot }}$, for three choices of the isotherm temperature $\theta: 0.1,0.5$ and 0.9 (a.u.), together with the transferred heat into a square of the same surface. The normalization ensures that all the curves approach the same saturation level. Several comments are in order: (i) The heat transferred by the irregular radiator is larger than that by the square. (ii) The normalized trans$T=0.9$. The agreement between numerical simulations and the experiment can be considered as good. This consideration is reinforced by the fact that taking care of the scale of temperature (40 to 18 centigrade) the value 0.9 correspond to a temperature of 37.8 centigrade while the isotherm color (yellow) is calibrated for 37 centigrade. (vi) The experiment does not allow a good determination of the short-time regime because the heat propagation was faster than the time to set the camera so that the earlier times could not be monitored.

In summary, the heat transfer in an irregularly-shaped domain with a prefractal geometry has been studied experimentally and numerically. The temperature evolution has been visualized experimentally by monitoring isocolor/isothermal lines. The area of a diffusion layer between the irregular heat source and a chosen isothermal line has been computed by thresholding the sequence of recorded images. We have shown that the de Gennes' suggestion to describe the diffusion layer by a Minkowski sausage and to use its area as a proxy of the total amount of heat transferred up to time $t$ applies only to temperatures far from that of the heat source. For temperatures close to the heat source, confinement effects play an important role so that the temperature spatial distribution is very different from a Minkowski layer.

We have then shown in a particular prefractal case that irregular morphology significantly speeds up heat transfer. The ideas and concepts developed in this study apply to any irregular shape, fractal or not. This suggests that irregular or fractal shapes can be used to increase heat removal from pulsed heat sources in electronics and microelectronic devices. Depending on temperature, the phenomenon may or may not be described by the con- 
cept of a Minkowski layer. More generally, these results apply to all physical phenomena governed by the Fourier equation, such as particle diffusion, spin diffusion and diffusion regimes in electrochemistry.

\section{Acknowledgments}

The authors acknowledge the contribution by Janos Kertesz who participated in the early stages of the experiment.

* Electronic address: anna.rozanova-pierrat@ecp.fr

† Electronic address: denis.grebenkov@polytechnique.edu

¥ Electronic address: bernard.sapoval@polytechnique.edu

[1] N. G. Makarov, Proc. Lond. Math. Soc. 51, 369 (1985).

[2] T. C. Halsey and M. Leibig, Ann. Phys. 219, 109 (1992).

[3] B. Sapoval, Phys. Rev. Lett. 73, 3314 (1994).

[4] D. S. Grebenkov, M. Filoche and B. Sapoval, Phys. Rev. E 73, 021103 (2006).

[5] T. Pajkossy and L. Nyikos, Electrochimica Acta 34, 171179 (1989); 34, 181-186 (1989).

[6] B. Sapoval, in "Fractals and disordered systems" ed. by A. Bunde, S. Havlin, pp. 207-225 (Springer-Verlag, 1991).

[7] B. Sapoval, S. Russ, J.-P. Korb, and D. Petit, Fractals 4, 453-462 (1996).

[8] D. S. Grebenkov, Rev. Mod. Phys. 79, 1077-1137 (2007).

[9] P. Levitz, D. S. Grebenkov, M. Zinsmeister, K. M. Kolwankar, and B. Sapoval, Phys. Rev. Lett. 96, 180601 (2006).

[10] D. Vandembroucq, C. Boccara, and S. Roux, Europhys. Lett., 30 (4), 209-214 (1995).
[11] C. G. Phillips and K. M. Jansons, Proc. Royal Soc. London A 428, 431-449 (1990).

[12] J. Fleckinger, M. Levitin, and D. Vassiliev, Proc. London Math. Soc. 71, 372-396 (1995).

[13] M. Levitin and D. Vassiliev, Proc. London Math. Soc. 72, 188-214 (1996).

[14] M. van den Berg and F. den Hollander, Proc. London Math. Soc. 78, 627-661 (1999).

[15] M. van den Berg, Probab. Theory Relat. Fields 118, 17$36(2000)$.

[16] D. Campos, V. Mendez, and J. Fort, Phys. Rev. E 69, 031115 (2004).

[17] M. R. Lancia, Le Matematiche 67, 271-287 (2007).

[18] B. Mandelbrot, The Fractal Geometry of Nature (Freeman \& Company, New York, 1982).

[19] M. Filoche and B. Sapoval, "Transfer Across Random versus Deterministic Fractal Interfaces", Phys. Rev. Lett. 84, 5776 (2000).

[20] P.-G. de Gennes, C. R. Acad. Sc., série II 295, 1061 (1982).

[21] A. Imre, T. Pajkossy and L. Nyikos, Acta Metallurgica Mater. 40, 1819-1826 (1992).

[22] J. Stasiek, Heat Mass Transf. 33, 27-39 (1997).

[23] C. R. Smith, D. R. Sabatino and T. J. Praisner, Exper. Fluids 30, 190-201 (2001).

[24] J. Crank, The Mathematics of Diffusion, 2nd Ed. (Clarendon, Oxford, 1975).

[25] H. S. Carslaw and J. C. Jaeger, Conduction of Heat in Solids, 2nd Ed. (Clarendon, Oxford, 1959).

[26] M. Schroeder, Fractals, Chaos, Power Laws (Freeman \& Company, New York, 1991).

[27] The Minkowski sausage (or $\epsilon$-neighboring) $\Omega_{\epsilon}$ of an arbitrary set $\Omega$ is the set of points within a distance $\varepsilon$ to this set [18, 26]. Although this term is sometimes used for the quadratic von Koch curve, this identification is erroneous and misleading. 